\title{
Sinus lift sans utilisation de substitut osseux : évaluation radiologique sur 28 patients traités avec 35 implants. Etude rétrospective sur 3 ans
}

\author{
Nicolas Strube \\ Cabinet de Chirurgie buccale, 13 rue de l'Arche sèche, 44000 Nantes, France \\ chir.strube@gmail.com
}

La réhabilitation des secteurs maxillaires postérieurs nécessite le plus souvent une augmentation du volume osseux. L'utilisation de biomatériaux semble nécessaire pour obtenir des résultats prédictibles.

Dans un souci de simplification, certains préconisent l'utilisation d'implants courts, d'autres longent les parois sinusiennes (Renouard 2005, Malo 2010, Anitua 2010). Le souhait de chacun est de diminuer les complications et la morbidité dues aux sinus lifts (Esposito 2010).

De récentes études (Lundgren 2004, Thor 2007, Fermergard 2009, Cricchio 2011, Volpe 2011) proposent une simplification des protocoles avec la suppression de l'utilisation de biomatériaux et publient des résultats encourageants.

Ojectifs: Evaluation radiologique de la formation osseuse entourant les implants insérés dans l'espace créé par l'élévation de la membrane de Schneider sans ajout de substitut osseux.

Matériel et méthodes: L'étude porte sur 28 patients édentés partiels ou unitaires, présentant une hauteur d'os résiduelle comprise entre 2,5 et $9 \mathrm{~mm} .37$ implants AstraTech ${ }^{\circledR}$ cylindro-coniques de 9 à $13 \mathrm{~mm}$ furent posés (dans 28 sinus) entre janvier 2009 et septembre 2011 avec réalisation d'un sinus lift sans utilisation de matériaux de comblement osseux. La hauteur intra-sinusienne des implants est mesurée radiologiquement à T0, puis lors du deuxième temps chirurgical (T0 + 4 à 6 mois), puis annuellement pour évaluer le gain osseux intra-sinusien.

Résultats: Tous les implants sont stables et ostéointégrés lors de la visite de contrôle (T0 + 4 à 6 mois). L'analyse radiologique montre une formation osseuse péri-implantaire dans 78,7\% des cas à 6 mois. Le gain osseux intra-sinusien est de $5,81 \mathrm{~mm}+/-2,22 \mathrm{~mm}$ après 6 mois ou plus.

Conclusion: L'élévation de la membrane de Schneider et le placement immédiat d'un implant sans utilisation de substitut osseux semble être une technique fiable avec des résultats prédictibles. Ce protocole pourrait réduire la morbidité associée à l'utilisation de biomatériaux. Des études histologiques demeurent toutefois nécessaires afin de confirmer la nature et l'architecture du tissu radio-opaque formé. 